\title{
MODULI OF ENDOMORPHISMS OF SEMISTABLE VECTOR BUNDLES OVER A COMPACT RIEMANN SURFACE
}

\author{
by L. BRAMBILA PAZ
}

(Received 6 November, 1985; revised 26 October, 1987 and 24 February, 1989)

Introduction. Mumford and Suominen in [8] and Newstead in [11] have considered the moduli problem of classifying the endomorphisms of finite-dimensional vector spaces. Using similar ideas we consider the moduli problem for endomorphisms of indecomposable semistable vector bundles over a compact connected Riemann surface of genus $g \geq 2$.

In this paper we develop the 3-dimensional case, which gives an idea of how to solve the moduli problem in general. First we give the algebras which can occur as algebras of endomorphisms of vector bundles of rank 3 . Then we give necessary and sufficient conditions for a vector bundle to have a particular algebra of endomorphisms. Such conditions show that for any non-zero nilpotent endomorphism, there are extensions of vector bundles from which it can be reconstructed. Thus the problem of parametrizing endomorphisms is largely reduced to one of parametrizing extensions. We construct the corresponding universal families of extensions; unfortunately, this is not quite sufficient for us to obtain moduli spaces for endomorphisms themselves. However, in some cases we can obtain local universal families of endomorphisms. The algebra of endomorphisms depends on how the extensions are related.

In Section 1 we state the moduli problem for endomorphisms of vector bundles. In Section 2 we recall from [3] the relations between the extensions and the algebras of endomorphisms. Section 3 contains the constructions of the universal families of extensions which partially solve the moduli problem.

1. Moduli of endomorphisms. Throughout this paper $X$ will denote a compact connected Riemann surface of genus greater than 1 , and $S(n, d)$ the set of (isomorphism classes of) indecomposable semistable non-simple vector bundles of rank $n$ and slope $d$ over $X$.

Let $P(n, d)$ be the set of pairs $(E, \phi)$ where $E$ is in $S(n, d)$ and $\phi: E \rightarrow E$ is an endomorphism of vector bundles. We say that two pairs $(E, \phi)$ and $(F, \psi)$ are equivalent, written $(E, \phi) \sim(F, \psi)$, if there exists an isomorphism $\alpha: E \rightarrow F$ such that $\psi \circ \alpha=\alpha \circ \phi$.

By a family of endomorphisms parametrized by a variety $S$ we shall mean a pair $(E, \Phi)$, where $E$ is a vector bundle over $X \times S$ and $\Phi$ an endomorphism of $E$, such that for each $s \in S$ the restriction $(E, \Phi)_{X \times s}$ is in $P(n, d)$. Two families $(E, \Phi)$ and $(F, \Psi)$ parametrized by $S$ are equivalent if and only if the restrictions $(E, \Phi)_{X \times s}$ and $(F, \Psi)_{X \times s}$ are equivalent for each $s \in S$. Given a family of endomorphisms $(E, \Phi)$ parametrized by $S$ and a morphism $h^{\prime}: T \rightarrow S$, the family $h^{*}(E, \Phi)=\left(h^{*} E, h^{*} \Phi\right)$, where $h=i d_{X} \times h^{\prime}$, is called the induced family of endomorphisms.

DefintTion 1. A fine moduli space for $P(n, d)$ is a variety $M$ and a family of endomorphisms $(U, \Phi)$ parametrized by $M$, such that for any family of endomorphisms

Glasgow Math. J. 32 (1990) 1-12. 
$(W, \Psi)$ parametrized by a variety $S$ there exists a unique morphism $h^{\prime}: S \rightarrow M$ such that the induced family is equivalent to the family $(W, \Psi)$.

For any indecomposable vector bundle $E$ over $X$ the algebra of endomorphisms is a special algebra i.e. $\operatorname{END}(E) \cong(1) \oplus \mathrm{Nil}(E)$ (see [1]). Since any endomorphism is the sum of a scalar multiple of the identity and a nilpotent endomorphism we shall concentrate on the study of non-zero nilpotent endomorphisms.

2. Algebras of endomorphisms. Let $E$ be a vector bundle over $X$ of rank $n$. If $E$ is semistable then for any $x \in X$ the map $e_{x}: \operatorname{End}(E) \rightarrow \operatorname{End}\left(E_{x}\right)$ defined as $\phi \mapsto \phi_{x}$ is injective.

If $E$ is also indecomposable the image $e_{x}(\operatorname{END}(E))=G(E)$ is a special algebra in $M_{n \times n}(\mathbb{C})$. Denote by $N(E)$ the subalgebra $e_{x}(\operatorname{Nil}(E))$. Let $H$ be the set of all non-zero subspaces $W \subset E_{x}$ which are invariant under $G(E)$, i.e. $\phi(W) \subset W$ for all $\phi$ in $G(E)$. Let $V$ be a minimal subspace of $H$. For any $v \in V$, define $N(v) \subset V$ as the vector space $\left\{w \in E_{x} \mid \phi(v)=w\right.$ for some $\phi$ in $\left.N(E)\right\}$. From the minimal property of $V$ we see that $N(v)=0$ or $N(v)=V$. If $N(v)=V$ then there is a nilpotent element $\psi \in N(E)$ such that $\psi(v)=v$, which is a contradiction. Hence $N(v)=0$ and so $v$ is a common eigenvector for $G(E)$. By inductive procedure we can see that $E_{x}$ has a flag invariant under $G(E)$. The existence of such flag implies that one can choose a basis of $E_{x}$ such that, for all $\phi_{x}$ in $G(E), \phi_{x}$ is an upper triangular matrix with all its diagonal entries equal.

Hence we have the following proposition.

Proposition 1. If $E$ is in $S(n, d)$ then $\operatorname{dim} \operatorname{END}(E) \leq 1+\frac{1}{2} n(n-1)$.

One question is: which special algebras, i.e. local rings, with fixed dimension can occur as algebras of endomorphisms of vector bundles in $S(n, d)$ ? In [3] we prove that for $E$ in $S(3, d), \operatorname{dim} \operatorname{END}(E) \neq 4$. Actually, $\operatorname{END}(E)$ is isomorphic to one of the algebras $\mathbb{C}[t] /\left(t^{2}\right), \mathbb{C}[t] /\left(t^{3}\right)$ or $\mathbb{C}[r, s] /(r, s)^{2}$.

If $E$ is in $S(3, d)$ and non-simple then there is a nilpotent endomorphism $\phi: E \rightarrow E$ such that $\phi^{2}=0$ and $\phi \neq 0$. Denote by $E_{2}$ the kernel of $\phi$ and by $L$ the image. Since $E$ is semistable and $\phi^{2}=0, E_{2}$ and $L$ are vector bundles over $X$ and define the exact sequences

$$
\xi: 0 \rightarrow E_{2} \stackrel{j}{\rightarrow} E \stackrel{\pi}{\rightarrow} L \rightarrow 0 \text { and } \rho: 0 \rightarrow L \stackrel{i}{\rightarrow} E_{2} \stackrel{p}{\rightarrow} L^{\prime} \rightarrow 0
$$

where $j \circ i \circ \pi \sim \phi$.

The type of algebra of endomorphisms depends on the relation between the extensions $\xi$ and $\rho$ and on whether $L^{\prime}$ is isomorphic to $L$ or not.

REMARK 1. In [3] we proved the following results.

(i) If $L \neq L^{\prime}$ then $\operatorname{END}(E) \cong \mathbb{C}[t] /\left(t^{2}\right)$.

(ii) If $L \cong L^{\prime}$ and $\rho=0$ then $\operatorname{END}(E) \cong \mathbb{E}[r, s] /(r, s)^{2}$.

(iii) If $L \cong L^{\prime}$ and $\rho \neq 0$ then from the surjective homomorphism $p_{*}: \operatorname{Ext}\left(L, E_{2}\right) \rightarrow$ $\operatorname{Ext}(L, L)$ we see that:

(1) if $p_{*}(\xi)=0$ then $\operatorname{END}(E) \cong \mathbb{C}[r, s] /(r, s)^{2}$;

(2) if $p_{*}(\xi)=\lambda \rho$ for some $\lambda \in \mathbb{C}^{*}$ then $\operatorname{END}(E) \cong \mathbb{C}[t] /\left(t^{3}\right)$;

(3) if $p_{*}(\xi) \neq \lambda \rho$ for $\lambda \in \mathbb{C}$ then $\operatorname{END}(E) \cong \mathbb{C}[t] /\left(t^{2}\right)$. 
Denote by $\Omega^{1}, \Omega^{2}$ and $\Omega^{3}$ the subsets of $S(3,0)$ of those vector bundles satisfying conditions (1), (2) and (3) respectively.

If $p_{*}(\xi)=\lambda \rho$ for some $\lambda \in \mathbb{C}^{*}$ then we have the following diagram

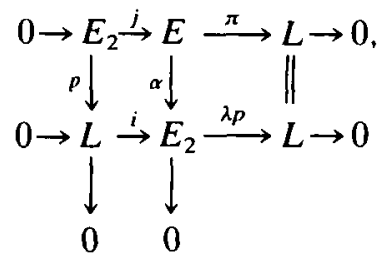

which can be completed as follows.

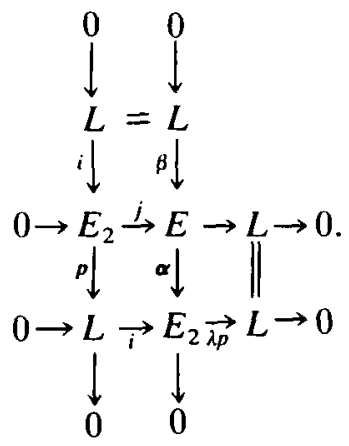

In this case, the composition $j \circ \alpha$ is a nilpotent endomorphism $\psi$ such that $\psi^{3}=0$ and $\psi^{2}$ is equivalent to $\lambda^{-1} \phi$.

Now let $L, L^{\prime}$ be two line bundles with the same slope, and $\rho: 0 \rightarrow L \stackrel{i}{\rightarrow} F \stackrel{p}{\rightarrow} L^{\prime} \rightarrow 0$ an extension of $L^{\prime}$ by $L$. If $\xi: 0 \rightarrow F^{\prime} \rightarrow E \stackrel{\pi}{\rightarrow} L \rightarrow 0$ is an extension of $L$ by $F$ then

(i) $E$ is semistable;

(ii) the pair of extensions $(\xi, \rho)$ defines a nilpotent endomorphism $(j \circ i \circ \pi)=\phi$ of index 2 , i.e. $\phi \neq 0$ but $\phi^{2}=0$;

(iii) if $\xi$ and $\rho$ are non-trivial then $E$ is indecomposable;

(iv) if $\rho$ is trivial then $E$ is indecomposable if and only if either

(a) $L \neq L^{\prime}$ and $\xi$ is not in either of the subspaces $\operatorname{Ext}(L, L)$ or $\operatorname{Ext}\left(L, L^{\prime}\right)$ of $\operatorname{Ext}(L, F)=\operatorname{Ext}\left(L, L \oplus L^{\prime}\right)$, or

(b) $L \cong L^{\prime}$ and $\xi$ is not in the image of $i_{*}: \operatorname{Ext}(L, L) \rightarrow \operatorname{Ext}(L, F)=$ $\operatorname{Ext}(L, L \oplus L)$ for any of the inclusions $i: L \rightarrow L \oplus L$.

If $L \cong L^{\prime}$ denote by $S(L)$ the image of such inclusions.

With a pair $(\xi, \rho)$ of extensions as above we obtain a pair $(E, \phi)$ in $P(3, d)$.

Denote by $E(3, d)$ the set of pairs of extensions $(\xi, \rho)$ as above.

Remark 2. (i) Note the pairs $(\xi, \rho)$ and $(\xi, \lambda \rho)$ define the same nilpotent endomorphism of index 2. Moreover, if $L=L^{\prime}$ and $\alpha=1+\mu i p$ is an automorphism of $F$ then, in general, the extension $\alpha \xi: 0 \rightarrow F \stackrel{j \alpha}{\rightarrow} E \stackrel{\pi}{\rightarrow} L \rightarrow 0$ is not equivalent to $\xi$, but the corresponding endomorphisms $j i \pi$ and joi $\pi$ are identical. 
(ii) If $\alpha$ extends to an automorphism of $E$, the extension $\alpha \xi$ is equivalent to $\xi$, so this problem does not arise. In particular, there is no problem when all automorphisms of $F$ extend to $E$, which happens

(a) when $F$ is simple, i.e. $L \neq L^{\prime}$, and $\rho$ non-trivial,

(b) in the case $L \cong L^{\prime}, \rho$ non-trivial, precisely when $E$ belongs to $\Omega^{1}$ or $\Omega^{2}$.

To study the moduli problem for endomorphisms we split $P(3, d)$ as follows:

$$
\begin{aligned}
& P^{0}=\left\{(E, \phi) \in P(3, d): \phi^{3}=0 \text { but } \phi^{2} \neq 0\right\}, \\
& P^{1}=\left\{(E, \phi) \in P(3, d): L \cong L^{\prime} \text { and } E_{2} \neq L \oplus L^{\prime}\right\}, \\
& P^{2}=\left\{(E, \phi) \in P(3, d): L \neq L^{\prime} \text { and } E_{2} \neq L \oplus L^{\prime}\right\}, \\
& P^{3}=\left\{(E, \phi) \in P(3, d): L \neq L^{\prime} \text { and } E_{2} \cong L \oplus L^{\prime}\right\}, \\
& P^{4}=\left\{(E, \phi) \in P(3, d): L \cong L^{\prime} \text { and } E_{2} \cong L \oplus L^{\prime}\right\},
\end{aligned}
$$

where as before, $E_{2}$ denotes the kernel of $\phi, L$ is the image of $\phi$ and $L^{\prime}=E_{2} / L$.

Without loss of generality we assume that $d=0$.

For each set $P^{i}$ we assign a set $P_{i}$ of equivalence classes of pairs of extensions in $E(3, d)$. The equivalence relation on the pairs of extensions depends on each of the sets $P^{i}$, so we shall treat them differently.

3. Moduli spaces. We split this section into five parts. In each one we construct a universal family of extensions and show how these provide a partial solution to the moduli problem for endomorphisms.

Let $E$ and $F$ be two families of vector bundles over $X$ parametrized by a variety $S$ such that $\operatorname{dim} H^{i}\left(X, \operatorname{Hom}\left(E_{s}, F_{s}\right)\right)$ is independent of $s \in S$ for $i=0,1$. Let $p: X \times S \rightarrow S$ be the projection and let us denote the vector bundle $R_{p}^{1}(\operatorname{Hom}(E, F))$ by $V$. Hence, we have the commutative diagram

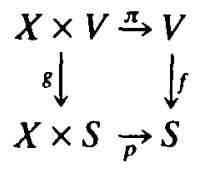

Lange in [7], using Grothendieck's universal properties of vector bundles, proved that if

$$
H^{i}\left(S, R_{p}^{0} \operatorname{Hom}(E, F) \otimes R_{p}^{1} \operatorname{Hom}(E, F)^{*}\right)=0
$$

for $i=1,2$ then $H^{1}\left(X \times S, \operatorname{Hom}(E, F) \otimes p^{*} R_{p}^{1}(\operatorname{Hom}(E, F))^{*}\right) \cong \operatorname{END}(V)$ and the identity in $\operatorname{END}(V)$ induces a universal family of extensions

$$
\Omega: 0 \rightarrow g^{*} F \rightarrow Z \rightarrow g^{*} E \rightarrow 0
$$

of $E$ by $F$ parametrized by $V$ such that $\Omega_{v}$ is the extension represented by $v$, for each $v \in V$. Moreover, if $\mathbb{P}(V)$ is the projective bundle associated to $V$ then (see Corollary 4.5 in [7]) there exists a universal family

$$
P \Omega: 0 \rightarrow g^{*} F \otimes p^{*} O_{\mathbb{P}(V)}(\mathbf{1}) \rightarrow \tilde{Z} \rightarrow g^{*} E \rightarrow 0 .
$$

of extensions over $X \times \mathbb{P}(V)$ which parametrizes all the classes of non-splitting extensions of $E_{s}$ by $F_{s}$ over $X$ modula the equivalence relation of identifying extensions which differ 
by a non-zero constant. From the universal properties of $\Omega$ and $P \Omega$ and the canonical map $\pi: V-\{0\} \rightarrow \mathbb{P}(V)$ we see that $\left(i d_{x} \times \pi\right)^{*} \mathbb{P}(V)$ is equivalent to $\Omega_{\mid V-\{0\}}$.

We shall prove that for some cases such universal extensions exist.

I. Let $P_{1}$ be the set of equivalence classes of pairs $(\xi, \rho)$, where $\rho \neq 0$ and $L \cong L^{\prime}$. Two pairs $(\xi, \rho)$ and $\left(\xi^{\prime}, \rho^{\prime}\right)$ are equivalent if and only if $\xi=\xi^{\prime}$ and $\rho=\lambda \rho^{\prime}$ for some $\lambda$ in $\mathbb{C}^{*}$. We shall construct a moduli space for $P_{1}$.

Let $T$ be the vector space $\operatorname{Ext}_{X}(1,1) \cong H^{1}(X, 1)$ and denote by $\mathbb{P}(T)$ the projective space of $T$. If $\mathbb{H}$ is the hyperplane bundle over $\mathbb{P}(T)$ then there exists a universal extension

$$
P \beta: 0 \rightarrow p^{*} \mathbb{R} \rightarrow W \rightarrow 1 \rightarrow 0
$$

over $X \times \mathbb{P}(T)$ that parametrizes all classes of non-splitting extensions of 1 by $\mathbf{1}$, modulo the equivalence relation of identifying extensions which differ by a non-zero constant, (see [9, Lemma 2.3]).

Let us consider the families of vector bundles $p^{*} \mathbb{H}$ and $W$ over $X \times \mathbb{P}(T)$. We recall from [4] the proof that

$$
H^{i}\left(\mathbb{P}(T), R_{p}^{0} \operatorname{Hom}\left(p^{*} \mathfrak{H}, W\right) \otimes R_{p}^{1} \operatorname{Hom}\left(p^{*} \mathfrak{H}, W\right)^{*}\right)=0
$$

for $i=1,2$. Basically we need the following lemmas.

LEMMA $1 . R_{p}^{0}(W) \cong \mapsto$.

Proof. From the exact sequence (1) we hàve the exact sequence

$$
\mathbf{0} \rightarrow \mathbf{1} \rightarrow W \otimes p^{*} \mathbb{H}^{*} \rightarrow p^{*} \mathbb{H}^{*} \rightarrow 0
$$

of vector bundles over $X \times \mathbb{P}(T)$, which induces the following exact sequence

$$
\begin{aligned}
0 & \rightarrow 1 \stackrel{f}{\rightarrow} p_{*} W \otimes \mathbb{H}^{*} \rightarrow \mathbb{H}^{*} \stackrel{\partial}{\rightarrow} H^{1}(X, 1) \otimes_{\mathbb{P}(T)} \odot \stackrel{g}{\rightarrow} R_{p}^{1}(W) \otimes H^{*} \\
& \rightarrow H^{1}(X, 1) \otimes_{\mathbb{P}(T)} \odot \otimes \mathbb{H}^{*} \rightarrow 0
\end{aligned}
$$

over $\mathbb{P}(T)$. Since $\operatorname{dim} H^{0}\left(X, W_{t}\right)=1$ for all $t \in \mathbb{P}(T)$, we see that $p_{*} W \otimes \mathbb{H}^{*}$ is a line bundle and hence the inclusion $f: 1 \rightarrow p_{*} W \otimes \mathbb{H}^{*}$ is an isomorphism, so that $p_{*} W$ is isomorphic to $\mathbb{H}$, which proves the Lemma.

Since the map $f: \mathbf{1} \rightarrow p_{*} W \otimes H^{*}$ is an isomorphism we have from the exact sequence (2) the following exact sequence

$$
0 \rightarrow \mathbb{H}^{*} \stackrel{\partial}{\rightarrow} H^{1}(X, 1) \otimes_{\mathbb{P}(T)} \stackrel{\circ}{\circ} \stackrel{g}{\rightarrow} R_{p}^{1}(W) \otimes \mathbb{H}^{*} \rightarrow H^{1}(X, 1) \otimes_{\mathbb{P}(T)} \mathcal{O} \otimes \mathbb{H}^{*} \rightarrow 0,
$$

which we split in two, namely

and

$$
0 \rightarrow H^{*} \stackrel{\partial}{\rightarrow} H^{1}(X, 1) \otimes_{\mathbb{P}(T)} \mathcal{O} \rightarrow I_{B} \rightarrow 0
$$

$$
0 \rightarrow I_{g} \rightarrow R_{p}^{1}(W) \otimes \mathbb{H}^{*} \rightarrow H^{1}(X, 1) \otimes_{P(T)} \odot \otimes \mathbb{H}^{*} \rightarrow 0,
$$

where $I_{g}$ is the image vector bundle. Since $g$ is a homomorphism of vector bundles of constant rank, $I_{g}^{*}$ is a vector bundle over $\mathbb{P}(T)$. We take the dual sequences

and

$$
0 \rightarrow I_{g}^{*} \rightarrow H^{1}(X, 1)^{*} \otimes_{\mathbb{P}(T)} \stackrel{\circ}{\rightarrow} \mathbb{H} \rightarrow 0
$$

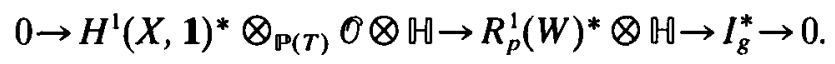


From the exact sequence (4) we see that:

LEMma 2. $H^{i}\left(\mathbb{P}(T), R_{p}^{1}(W)^{*} \otimes \mathbb{H}\right) \cong H^{i}\left(\mathbb{P}(T), I_{g}^{*}\right)$ for $i \geq 1$.

Proof. The lemma follows from the equality $H^{i}(\mathbb{P}(T), \mathbb{H}) \otimes H^{1}(X, 1)^{*}=0$ for all $i \geq 1$ and the cohomology sequence associated to the exact sequence (4).

LEMMA 3. If $\partial: H^{1}(X, 1)^{*} \bigotimes_{\mathbb{P}(T)} \mathscr{O} \rightarrow \mathbb{H}$ is the surjective homomorphism in (3) then the induced map $\partial^{*}: H^{0}(\mathbb{P}(T), \mathcal{O}) \otimes H^{1}(X, 1)^{*} \rightarrow H^{1}(\mathbb{P}(T)$, N) is an isomorphism.

Proof. The map $\partial$ coincides with the tautological surjection $T^{*} \times \mathbb{P}(T) \rightarrow \mathbb{H}$. It is a standard fact (following easily from the definition of $\mathbb{H}$ ) that this induces an isomorphism of spaces of sections.

Lemma 4. $H^{i}\left(\mathbb{P}(T), I_{g}^{*}\right)=0$ for $i \geq 0$.

Proof. Part of the cohomology sequence of (3) is

$$
\rightarrow H^{i}(\mathbb{P}(T), \mathbb{H}) \rightarrow H^{i+1}\left(\mathbb{P}(T), I_{g}^{*}\right) \rightarrow H^{i+1}(\mathbb{P}(T), \mathcal{O}) \otimes H^{1}(X, 1)^{*} \rightarrow
$$

Now $H^{i}(\mathbb{P}(T), \mathbb{H})=0=H^{i}(\mathbb{P}(T), \mathcal{O})$ for $i \geq 1$, so $H^{i}\left(\mathbb{P}(T), I_{g}^{*}\right)=0$ for $i \geq 2$. Thus we have the exact sequence

$$
0 \rightarrow H^{0}\left(\mathbb{P}(T), I_{g}^{*}\right) \rightarrow H^{0}(\mathbb{P}(T), \mathcal{O}) \otimes H^{1}(X, 1)^{*} \stackrel{\partial}{\rightarrow} H^{0}(\mathbb{P}(T), \mathbb{H}) \rightarrow H^{1}\left(\mathbb{P}(T), I_{g}^{*}\right) \rightarrow 0
$$
$i=0,1$.

But from Lemma 3 we know that $\partial$ is an isomorphism, hence $H^{i}\left(\mathbb{P}(T), I_{g}^{*}\right)=0$ for

Proposition 2. $H^{i}\left(\mathbb{P}(T), R_{p}^{0} \operatorname{Hom}\left(p^{*} \mathbb{H}, W\right) \otimes R_{p}^{1} \operatorname{Hom}\left(p^{*} \mathfrak{H}, W\right)^{*}\right)=0$ for $i=1,2$. and 4.

Proof. From Lemma $1, R_{p}^{0}(W) \cong \mathbb{H}$; hence the Proposition follows from Lemmas 2

THEOREM 1. There exists a fine moduli space for $P_{1}$.

Proof. From Lange's results there is a universal extension

$$
\Omega: 0 \rightarrow g^{*} W \stackrel{j}{\rightarrow} Z \rightarrow g^{*} p^{*}(\mathbb{H}) \rightarrow 0
$$

of vector bundles over $X \times V$, where $V$ is $R_{p}^{1} \operatorname{Hom}\left(p^{*} R, W\right)$, which parametrizes all extensions of 1 by $W_{t}$, for all $t \in \mathbb{P}(T)$. Let $\tilde{V}$ be the complement, in $V$, of the zero section $s_{0}: \mathbb{P}(T) \rightarrow R_{p}^{1} \operatorname{Hom}\left(p^{*} \mathbb{H}, W\right)$.

From the restrictions of the exact sequence $\Omega$ and the induced extension $P \beta$, we have over $X \times \tilde{V}$, the following exact sequences

and

$$
\Omega: 0 \rightarrow g^{*} W \stackrel{j}{\rightarrow} Z \stackrel{\pi}{\rightarrow} g^{*} p^{*}(\mathbb{H}) \rightarrow 0
$$

$$
g^{*} P \beta: 0 \rightarrow g^{*} p^{*} \stackrel{i}{\rightarrow} g^{*} W \stackrel{\pi}{\rightarrow} \mathbf{1} \rightarrow 0 .
$$

Let $M_{1}$ be $\bar{V} \times \operatorname{Pic}_{0}(X)$. From the universal properties of the extensions $P \beta$ and $\Omega$ we see that the pair of extensions $\left(\Omega, g^{*} P \beta\right)$ induces on $X \times M_{1}$ a pair of extensions which, after tensoring by the pull-back of the Poincaré bundle $L$, define the moduli space for $P_{1}$.

Let $(Z, \Phi)$ be the family of endomorphisms over $X \times \bar{V}$ given by $\Phi=j \circ i \circ \pi$. The restriction $Z_{X \times v}$ is in $S(3,0)$, for each $v \in \tilde{V}$. However we know, from Section 2, that not all the vector bundles $Z_{X \times v}$ have the same algebra of endomorphisms. 
If $V$ and $\mathbb{P}(T)$ are as above then the monomorphism $j: \mathbb{H}^{* 2} \rightarrow H^{1}(X, 1) \otimes_{\mathbb{P}(T)} \odot \otimes \mathbb{H}^{*}$ induces the commutative diagram

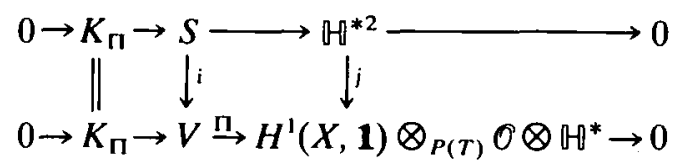

where $i$ is an inclusion. Hence $K_{\Pi}$ is a subbundle of $S$ and $\Pi(S)=\mathbb{H}^{* 2}$. This means that for each $s \in(S)_{t}, \Pi_{t}(s)=\lambda \beta_{t}$, where $\lambda \in \mathbb{C}^{*}$ and $\beta_{t} \in[t]$. Denote by $S^{1}$ the kernel $K_{\Pi}$, by $S^{2}$ the space $S-S^{1}$ and by $S^{3}$ the space $V-S$.

THEOREM 2. Let $Z$ be the family of vector bundles parametrized by $\tilde{V}$ given in Theorem 1. Then (i) $Z_{m} \in \Omega^{1}$ iff $m \in S^{1}$, (ii) $Z_{m} \in \Omega^{2}$ iff $m \in S^{2}$, (iii) $Z_{m} \in \Omega^{3}$ iff $m \in S^{3}$.

Proof. The Theorem follows from the definition of $\Omega^{i}$ and $S^{i}$.

Now let $\left(Z_{1}, \Phi\right)$ denote the family of endomorphisms over $X \times M_{1}$ induced by $(Z, \Phi)$. For $i=1,2,3$ let $P^{1 i}$ denote the set of the pairs $(E, \Phi) \in P^{1}$ such that $E \in \Omega^{i}$, let $M_{1 i}=\left(\tilde{V} \cap S^{i}\right) \times \operatorname{Pic}_{0}(X)$ and let $\left(Z_{1 i}, \Phi\right)$ denote the restriction of $\left(Z_{1}, \Phi\right)$ to $X \times M_{1 i}$. We deduce at once from Theorem 2 and Remark $2(b)$ the following result. for $P^{1 i}$

COROLlaRY. For $i=1,2$, the family of endomorphisms $\left(Z_{1 i}, \Phi\right)$ is a universal family

When $i=3$, the situation is more complicated (see Remark 2 again).

II. Denote by $P^{0}$ the set of (equivalence classes of) pairs $(E, \phi)$ in $P(3, d)$ such that $\phi^{3}=0$ but $\phi^{2} \neq 0$. For each pair $(E, \phi)$ in $P^{0}$ we have the following commutative diagram of vector bundles

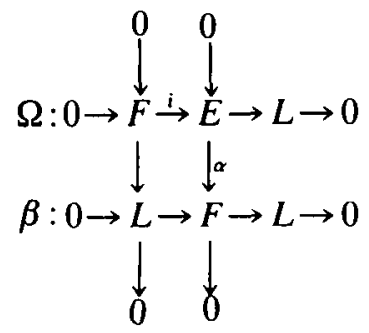

where $F$ and $L$ are the image and kernel of $\phi$ respectively. We consider the problem of parametrizing such diagrams up to equivalence of the extensions $\beta$ and $\Omega$. Denote by $P_{0}$ the set of equivalence classes of the diagrams.

A diagram as above defines a pair $(E, i \circ \alpha)$ in $P^{0}$. Two pairs $(E, \phi)$ and $\left(E, \phi^{\prime}\right)$ in $P^{0}$ define the same element in $P_{0}$ iff $\phi=(1+\psi) \phi^{\prime}$ with $\psi$ nilpotent. Again we solve the moduli problem for $\boldsymbol{P}_{\mathbf{0}}$.

Let $T_{0}$ be the space $T-\{0\}$, where $T \cong H^{1}(X, 1)$ and let

$$
\beta: 0 \rightarrow 1 \rightarrow W_{0} \stackrel{p}{\rightarrow} 1 \rightarrow 0
$$

be the extension over $X \times T_{0}$ that parametrizes all the non-trivial extensions of 1 by 1 (see [10]). Actually, if $h^{\prime}: T_{0} \rightarrow \mathbb{P}(T)$ is the natural map then $h^{*}=\left(i d_{X} \times h^{\prime}\right)^{*}(P \beta)=\beta$, where $P \beta$ is the extension (1). Moreover, $h^{*}(W)=W_{0}$. 
Let $\pi: X \times T_{0} \rightarrow T_{0}$ be the projection and denote by $V_{0}$ the vector bundle associated to $R_{\pi}^{1}\left(W_{0}\right)$. From the following commutative diagram

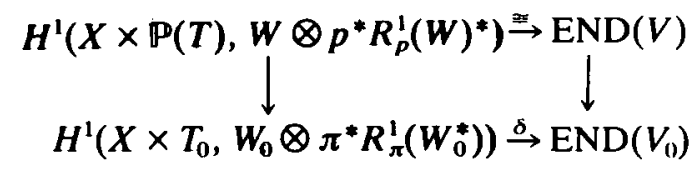

we have that the identity in $\operatorname{END}(V)$ defines an element $\beta_{0}$ in $H^{1}\left(X \times T_{0}, W_{0} \otimes \pi^{*} R_{\pi}^{1}\left(W_{0}\right)^{*}\right)$ such that $\delta\left(\beta_{0}\right)=i d_{V_{0}}$. If $g: X \times V_{0} \rightarrow X \times T_{0}$ is the induced homomorphism then the element $\beta_{0}$ defines under the following natural maps

$$
\begin{aligned}
H^{1}\left(X \times T_{0}, W_{0} \otimes \pi^{*} R_{\pi}^{1}(W)^{*}\right) & \stackrel{\ulcorner}{\rightarrow} H^{1}\left(X \times T_{0}, W_{0} \otimes g_{*} \mathcal{O}_{X \times V_{0}}\right) \\
& \cong H^{1}\left(X \times T_{0}, g_{*} g^{*} W_{0}\right) \\
& \cong H^{1}\left(X \times V_{0}, g^{*} W_{0}\right),
\end{aligned}
$$

a universal extension

$$
\Omega: 0 \rightarrow g^{*} W_{0} \rightarrow Z_{0} \rightarrow 1 \rightarrow 0
$$

over $X \times V_{0}$ which parametrizes all the extensions of the trivial line bundle 1 by $W_{t}$, where $W_{t}$ is a non-trivial extension of 1 by 1 . We shall prove that there is a variety $S_{0} \subset V_{0}$ which parametrizes the diagrams for which $\beta \otimes L^{*}$ corresponds to a point of $T_{0}$.

The image of the extension $\beta$ under the homomorphism $H^{1}\left(X \times T_{0}, 1\right) \stackrel{\partial}{\rightarrow}$ $H^{0}\left(T_{0}, R_{p}^{1} \operatorname{Hom}(1,1)\right)$ given in the Leray spectral sequence defines a nowhere-vanishing section $s=\partial(\beta)$. This has the property that for each $t \in T_{0}, s(t) \in H^{1}(X, 1)$ is precisely the extension represented by $t$. The surjective homomorphism $p: W_{0} \rightarrow 1$ over $X \times T_{0}$ induces a surjective homomorphism $R_{p}^{1} \operatorname{Hom}\left(1, W_{0}\right) \rightarrow R_{p}^{1} \operatorname{Hom}(1,1)$ of vector bundles over $T_{0}$. Let us denote by $S_{0}$ the subspace $p^{-1}\left(s\left(T_{0}\right)\right) \subset V_{0}$. We now have two extensions $\beta_{1}: 0 \rightarrow 1 \stackrel{i}{\rightarrow} g^{*} W_{0} \stackrel{p}{\rightarrow} 1 \rightarrow 0$ and $\Omega_{1}: 0 \rightarrow g^{*} W_{0} \rightarrow Z \rightarrow 1 \rightarrow 0$ which are the restrictions of $\beta$ and $\Omega$ to $X \times S_{0}$.

Let $s_{1}$ and $s_{2}$ be the images of $\Omega_{1}$ and $\beta_{1}$ respectively under the homomorphisms $H^{1}\left(X \times S_{0}, g^{*} W_{0}\right) \stackrel{f}{\rightarrow} H^{0}\left(S_{0}, R_{p}^{1}\left(g^{*} W_{0}\right)\right)$ and $H^{1}\left(X \times S_{0}, 1\right) \stackrel{h}{\rightarrow} H^{0}\left(S_{0}, R_{p}^{1}(1)\right)$ given in the Leray spectral sequences. The surjective homomorphism $p: g^{*} W_{0} \rightarrow 1$ induces homorphisms $p_{1}^{*}$ and $p_{2}^{*}$ such that the diagram

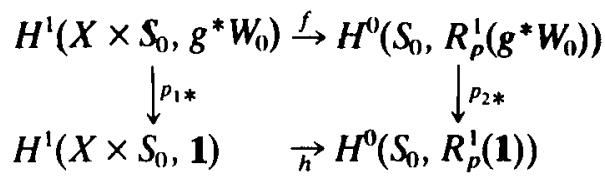

commutes.

From the definition of $S_{0}$, we see that $p_{2 *}\left(s_{1}\right)=s_{2}$, and so $p_{2 *}\left(f\left(\Omega_{1}\right)\right)=p_{2 *}\left(s_{1}\right)=s_{2}=$ $h\left(\beta_{\mid}\right)$. Hence, $h\left(p_{1 *}\left(\Omega_{1}\right)\right)=s_{1}=h\left(\beta_{1}\right)$. If $g \geq 3, H^{1}\left(T_{0}, 1\right)=0$, so $H^{1}\left(S_{0}, 1\right)=0$, the map $h$ is injective and hence $p_{1 *}\left(\Omega_{1}\right)$ and $\beta_{1}$ define the same family of extensions. The equality $p_{1 *}\left(\Omega_{1}\right)=\beta_{1}$ implies that there is a unique homomorphism $\alpha: Z \rightarrow g^{*}(W)$ such that the 
diagram

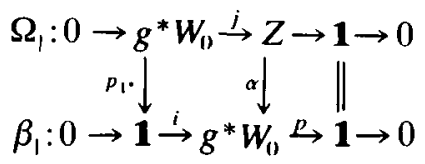

of vector bundles over $X \times S_{0}$ commutes. Such diagram can be completed as follows:

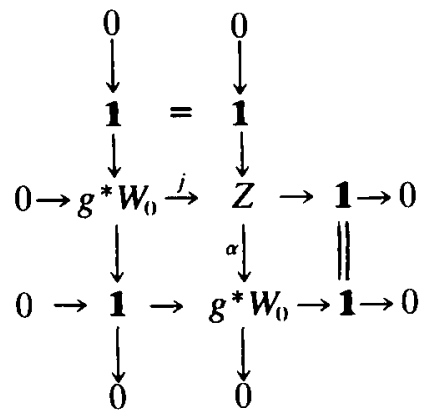

Let $M^{0}$ be $S_{0} \times \mathrm{Pic}_{0}(X)$ and consider the family of diagrams given by $\left(\pi_{12}^{*} \Omega_{1} \otimes \pi_{13}^{*} L, \pi_{12}^{*} \beta_{1} \otimes \pi_{13}^{*} L\right)$ over $X \times M_{0}$. This proves:

TheORem 3. If $g \geq 3$, there exists a fine moduli space for the diagrams in $P_{0}$ which lie over $S_{0} \times \operatorname{Pic}_{0}(X)$.

III. Let $P_{2}$ be the set of equivalence classes of pairs $(\xi, \rho)$ where $\rho \neq 0$ and $L \neq L^{\prime}$. Two pairs are equivalent iff $\rho=\lambda \rho^{\prime}$ for some $\lambda \in \mathbb{C}^{*}$ and $\xi=\xi^{\prime}$.

Denote by $\Delta^{c}$ the variety $\operatorname{Pic}_{0}(X) \times \operatorname{Pic}_{0}(X)-\Delta$, where $\Delta$ is the diagonal subvariety. If $p_{1 k}: X \times \Delta^{c} \rightarrow X \times \operatorname{Pic}_{0}(X)$ are the projections for $k=2,3$ then let $L_{k-1}$ be $p_{1 k}^{*} L$. Take the vector bundle $\operatorname{Hom}\left(L_{2}, L_{1}\right)$ over $X \times \Delta^{c}$. Since $R_{\pi_{2}}^{0}\left(\operatorname{Hom}\left(L_{2}, L_{1}\right)\right)=0$ we see from Lange's results that there is an extension

$$
\Omega: 0 \rightarrow \pi_{2}^{*} H \otimes g^{*} L_{1} \rightarrow W \rightarrow g^{*} L_{2} \rightarrow 0
$$

over $X \times \mathbb{P}(V)$, where $V$ is $R_{\pi_{2}}^{1} \operatorname{Hom}\left(L_{2}, L_{1}\right), g: X \times V \rightarrow X \times \Delta^{c}, \mathbb{P}(V)$ is the projective bundle of $V$ and $t-0$ the hyperplane bundle over $\mathbb{P}(V)$. Such an extension parametrizes all the classes of non-splitting extensions of two non-isomorphic line bundles with zero degree.

Let us take the vector bundle $\operatorname{Hom}\left(\pi_{2}^{*}\left[-\mathbb{D} \otimes g^{*} L_{1}, W\right)\right.$ over $X \times \mathbb{P}(V)$ and let $Z$ be the vector bundle $R_{\pi_{2}}^{1} \operatorname{Hom}\left(g^{*} L_{1}, W\right) \otimes \mathbb{H}^{*}$ over $\mathbb{P}(V)$. To use Lange's result we need to prove that

$$
H^{i}\left(\mathbb{P}(V), R_{\pi_{2}}^{0}\left(g^{*} L_{1}^{*} \otimes W\right) \otimes R_{\pi_{2}}^{1}\left(g^{*} L_{1}^{*} \otimes W\right)^{*}\right)=0
$$

for $i=1,2$ or that there exists a unique element in

$$
H^{1}\left(X \times \mathbb{P}(V), g^{*} L_{1}^{*} \otimes W \otimes \pi_{2}^{*} R_{\pi_{2}}^{1}\left(g^{*} L_{1}^{*} \otimes W\right)^{*}\right)
$$

which maps to the identity in $\operatorname{END}(Z)$.

To compute the cohomology groups we see that the exact sequence (7) induces the 
following exact sequence

$$
\begin{aligned}
0 \rightarrow \mathbb{W} & \rightarrow R_{\pi_{2}}^{0}\left(W \otimes g^{*} L_{1}^{*}\right) \rightarrow R_{\pi_{2}}^{0}\left(g^{*} L_{2} \otimes g^{*} L_{1}^{*}\right) \rightarrow R_{\pi_{2}}^{1}(1) \otimes \mathbb{N} \\
& \rightarrow R_{\pi_{2}}^{1}\left(W \otimes g^{*} L_{1}^{*}\right) \rightarrow R_{\pi_{2}}^{1}\left(g^{*} L_{2} \otimes g^{*} L_{1}^{*}\right) \rightarrow 0 .
\end{aligned}
$$

Since $R_{\pi_{2}}^{0}\left(g^{*} L_{2} \otimes g^{*} L_{1}^{*}\right)=0$, we have that $R_{\pi_{2}}^{0}\left(W \otimes g^{*} L_{1}^{*}\right) \cong \mathbb{H}$. Hence $R_{\pi_{2}}^{0}\left(g^{*} L_{1}^{*} \otimes W\right) \otimes R_{\pi_{2}}^{1}\left(g^{*} L_{1}^{*} \otimes W\right)^{*} \cong \mathbb{H} \otimes R_{\pi_{2}}^{1}\left(W \otimes g^{*} L^{*}\right)^{*}$. From the exact sequence (9) we obtain the exact sequence

$$
0 \rightarrow R_{\pi_{2}}^{1}\left(g^{*} L_{2} \otimes g^{*} L_{1}^{*}\right)^{*} \otimes \mathbb{H} \rightarrow R_{\pi_{2}}^{1}\left(W \otimes g^{*} L_{1}^{*}\right)^{*} \otimes \mathbb{H} \rightarrow R_{\pi_{2}}^{1}(1)^{*} \rightarrow 0 .
$$

Part of the cohomology sequence of $(10)$ is

$$
\begin{gathered}
H^{i}\left(\mathbb{P}(V), R_{\pi_{2}}^{1}\left(g^{*} L_{2} \otimes g^{*} L_{1}^{*}\right)^{*} \otimes \mathbb{H}\right) \rightarrow H^{i}\left(\mathbb{P}(V), R_{\pi_{2}}^{1}\left(W \otimes g^{*} L_{1}^{*}\right)^{*} \otimes \mathbb{H}\right) \\
\rightarrow H^{i}(\mathbb{P}(V), \mathcal{O}) \otimes H^{1}(X, \mathcal{O})^{*} \rightarrow .
\end{gathered}
$$

Since $\mathbb{P}(V)$ is a projective bundle over $\Delta^{c}$ we have that

$$
H^{i}(\mathbb{P}(V), \mathscr{O}) \cong H^{i}\left(\Delta^{c}, \mathcal{O}\right)
$$

and from the commutative diagram

we see that

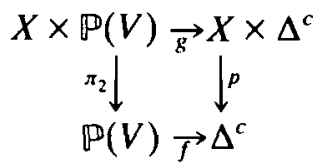

$$
H^{i}\left(\mathbb{P}(V), R_{\pi_{2}}^{1}\left(g^{*} L_{2} \otimes g^{*} L_{1}^{*}\right)^{*} \otimes \mathbb{M}\right)^{*} \cong H^{i}\left(\Delta^{c}, \sigma^{*} V^{*} \otimes V^{*}\right),
$$

where $\sigma$ is the canonical involution on $\Delta^{c}$.

In this case we arrive at the problem that we do not know if the cohomology groups (11) and (12) are zero or not, nor even if there exists a unique element which maps to the identity in $\operatorname{END}(Z)$.

Lange in [7] distinguishes between global families of extensions (the concept that we have been using) and families of extensions over a variety $M$ (see [7, page 105]). He defines "family of extensions" using an open cover of $M$. Over each open set there is a collection of extensions "glued" together to define an extension over the open set. If the covering may be taken to be $M$ itself, then the family is said to be "globally defined".

Using similar ideas we could introduce a more general definition of families of endomorphisms.

Definition. A family of endomorphisms parametrized by $M$ is a collection of pairs $\left\{(E, \phi)_{m}\right\}$ such that there exist

(i) an open cover $\left\{U_{\alpha}\right\}$ of $M$,

(ii) a vector bundle $W_{\alpha}$ and

(iii) an endomorphism $\phi_{\alpha}$ of $W_{\alpha}$ in each open set $U_{\alpha}$,

such that for each pair $(E, \phi)_{m}$ with $m \in U_{\alpha},\left(W_{\alpha}, \phi_{\alpha}\right)_{m}$ is equivalent to $(E, \phi)_{m}$. The family is said to be global if the open cover is $M$ itself. 
The global families of endomorphisms correspond to the concept of families of endomorphisms that we have been using (see Definition 1). If we use this new concept of family of endomorphisms (not global) then we can solve the moduli problem for $P^{2}$ as follows:

Recall from above, that $Z$ is a vector bundle over $P(V)$ and $V$ is a vector bundle over $\Delta^{c}$. Hence we can cover $P(V)$ by affine open sets $\left\{U_{\alpha}\right\}$ which induce a cover $\left\{V_{\alpha}\right\}$ over $Z$. The cohomology groups

$$
H^{1}\left(U_{\alpha}, R_{p}^{0}\left(\operatorname{Hom}\left(g^{*} L_{1}, W\right)\right) \otimes R_{p}^{1}\left(\operatorname{Hom}\left(g^{*} L_{1}, W\right)^{*}\right)\right)
$$

are zero for $i=1,2$, since $U_{\alpha}$ is affine.

Now we can apply Lange's results to prove that there is a universal extension of (the restrictions of) $g^{*}\left(L_{1}\right)$ by $W$ over $X \times V_{\alpha}$. Such an extension parametrizes all the extensions of a line bundle $L_{1}$ by $W_{t}$, where $W_{t}$ is in $U_{\alpha}$. Hence we have the following Theorem.

THEOREM 4. There exists a (local) universal extension for $P_{2}$.

In this case $W_{t}$ is simple, so by Remark 2(a) the extensions are determined by the endomorphisms. Hence

COROLLARY. There exists a (local) universal family of endomorphisms for $P^{2}$.

IV. Let $P_{3}$ be the set of all extensions $\xi: 0 \rightarrow L \oplus L^{\prime} \rightarrow E \rightarrow L \rightarrow 0$ with $L \neq L^{\prime}$ and $\mu(L)=\mu\left(L^{\prime}\right)=0$. As in the previous case take $\Delta^{c}$ and the line bundles $L_{1}$ and $L_{2}$ over $X \times \Delta^{c}$. If $p: X \times \Delta^{c} \rightarrow \Delta^{c}$ is the projection then the points in

$$
R_{p}^{1}\left(\operatorname{Hom}\left(g^{*}\left(L_{1}\right), g^{*}\left(L_{1} \oplus L_{2}\right)\right)\right)=V^{\prime}
$$

represent extensions in $P_{3}$.

To construct the (global) universal extension we must prove that the cohomology group $H^{i}\left(\Delta^{c}, V^{\prime}\right)$ is zero, for $i=1,2$.

As in the previous case, we only obtain a local universal extension, using a Stein cover of $\Delta^{c}$.

TheOREM 5. There exists a (local) universal family of extensions for $P_{3}$.

V. Let $P_{4}$ be the set of extensions $\xi: 0 \rightarrow L \oplus L \rightarrow E \rightarrow L \rightarrow 0$ such that $E$ is indecomposable.

If $R$ is the space $\operatorname{Ext}_{x}(1,1 \oplus 1)$ then there exists a universal extension

$$
0 \rightarrow \mathbf{1} \oplus \mathbf{1} \rightarrow Y \rightarrow \mathbf{1} \rightarrow 0
$$

over $X \times R$ that parametrizes all the extensions of 1 by $1 \oplus 1$ (see [10]). Take $S(1) \subset R$ as in Section 2 and denote $R-S(\mathbf{1})$ by $S_{0}$. If $M_{4}$ is $S_{0} \times \operatorname{Pic}_{0}(X)$ then the extension (13) induces over $X \times M_{4}$ the universal extension

$$
\xi: 0 \rightarrow L_{0} \oplus L_{0} \rightarrow Y_{0} \otimes L_{0} \rightarrow L_{0} \rightarrow 0
$$


where $L_{0}$ and $Y_{0}$ are the corresponding pull back of the Poincare bundle and the restriction of the vector bundle $Y$ to $X \times S_{0}$.

Thus, from the universal properties of the exact sequence (14) we have the following Theorem.

THEOREM 6. There is a fine moduli space for $P_{4}$.

Acknowledgments. I am most grateful to P. E. Newstead for many useful suggestions during the preparation of this paper.

\section{REFERENCES}

1. M. F. Atiyah, Complex analytic connections in fibre bundles, Trans, Amer. Math. Soc. 85 (1957), 181-207.

2. L. Brambila, Endomorphisms of vector bundles over a compact Riemann surface: 2-dimensional case, in Several complex variables (Pitman, 1985), 90-95.

3. L. Brambila, Algebras of endomorphisms of semistable vector bundles over a compact Riemann surface, Reportes de investigación 1986, Universidad Autónoma Metropolitana Mexico.

4. L. Brambila, Existence of universal extensions, preprint.

5. G. Harder and M. S. Narasimhan, On the cohomology group of moduli space of vector bundles on curves, Math. Ann. 212 (1975), 215-248.

6. R. Harstshorne, Algebraic geometry, (Springer Verlag, 1977).

7. H. Lange, Universal families of extensions, J. Algebra 83 (1983), 101-112.

8. D. Mumford and K. Suominen, Introduction to the theory of moduli, 5th Nordic Summer School in Math. Algebraic Geometry, Oslo, 1970.

9. M. S. Narasimham and S. Ramanan, Moduli of vector bundles on a compact Riemann surface, Ann. of Math. (2) 89 (1969), 14-51.

10. M. S. Narasimhan and C. S. Seshadri, Holomorphic vector bundles on a compact Riemann surface, Math. Ann. 155 (1964), 69-80.

11. P. E. Newstead, Lectures on introduction to moduli problems and orbit space (Tata Institute of Fundamental .Research, Bombay, 1978). 69-84.

12. S. Ramanan, Moduli of vector bundles over an algebraic curve, Math. Ann. 200 (1973),

13. S. S. Shatz, The decomposition and specialization of algebra families of vector bundles. Compositio Math. 35 (1977), 163-187.

Departamento de Matemáticas

Universidad Autónoma Metropolttana, Iztapalapa

Av. La Purisima y Michoacán, Iztapalapa, Apdo. Postal 55-534

MÉxico, D.F. C.P. 09340 